\title{
MIXED FINITE ELEMENT APPROXIMATION OF 3D CONTACT PROBLEMS WITH GIVEN FRICTION: ERROR ANALYSIS AND NUMERICAL REALIZATION
}

\author{
JAROSLAV HASLinger $^{1}$ AND TAOUfiK SASsi ${ }^{2}$
}

\begin{abstract}
This contribution deals with a mixed variational formulation of $3 \mathrm{D}$ contact problems with the simplest model involving friction. This formulation is based on a dualization of the set of admissible displacements and the regularization of the non-differentiable term. Displacements are approximated by piecewise linear elements while the respective dual variables by piecewise constant functions on a dual partition of the contact zone. The rate of convergence is established provided that the solution is smooth enough. The numerical realization of such problems will be discussed and results of a model example will be shown.
\end{abstract}

Mathematics Subject Classification. 65N30, 74M15.

Received: January 9, 2004.

\section{INTRODUCTION}

In the theory of variational inequalities, problems involving contact and friction conditions are of a permanent interest (we refer to [8] and to [18] for a large review of the main unilateral contact models). Finite elements are the most currently used methods for the approximation of contact problems involving friction (see [14, 17] and the references therein). In the primal variational formulation (where the displacement is the only unknown), discretized non-penetration conditions constitute the key point of the approximation model. These conditions can be relaxed and expressed in a weaker sense $[4,14,16]$. In the mixed variational formulation where the displacement and the normal stresses on the contact zone are treated as independent variables, the unilateral conditions have been expressed by using Lagrange multipliers (see [14]). Both variational formulations lead to a non-differentiable problem. To overcome this difficulty one can introduce (see [12]) another set of Lagrange multipliers related to the tangential component of the stress on the contact zone transforming the non-differentiable problem into a smooth one.

The purpose of this contribution is to extend this approach to $3 \mathrm{D}$ contact problems with given friction by using piecewise constant discretizations of Lagrange multipliers on the contact zone. Unlike to 2D problems, constraints imposed on the "tangential" Lagrange multipliers are now quadratic. This fact complicates not only

\footnotetext{
Keywords and phrases. Mixed finite element methods, unilateral contact problems with friction, a priori error estimates.

1 Department of Numerical Mathematics, Charles University 12116 Praha 2, Czech Republic. e-mail: mfkfk@met.mff.cuni.cz

${ }^{2}$ Laboratoire de Mathématiques Nicolas Oresme, CNRS UMR UFR Sciences Campus II, Bd Maréchal Juin, 14032 Caen Cedex, France. e-mail: sassi@math.unicaen.fr 
the theoretical analysis but also the practical realization. We establish a priori error estimates and propose the efficient numerical method.

The paper is organized as follows. Firstly, we introduce the mathematical model of the Signorini problem with given friction. In Section 2 we present the continuous mixed variational formulation of the problem. In the third section, we propose a well-posed finite element discretization in order to approximate the mixed formulation. In Section 4 a priori error estimates for the mixed finite element approximations are established. Finally, in Section 5 we present a numerical approach which is based on the dual variational formulation (i.e. the formulation in terms of the Lagrange multipliers) combined with a linearization of the quadratic constraints. Numerical results of a model example will be shown.

\section{Preliminary and nOtations}

The Euclidean norm of a point $\boldsymbol{x} \in \mathbb{R}^{n}, n \geq 2$ will be denoted $\|\boldsymbol{x}\|$ in what follows.

Let $\Omega \subset \mathbb{R}^{n}$ be a bounded domain with the Lipschitz boundary $\partial \Omega$. The symbol $H^{k}(\Omega), k \geq 1$ integer, stands for the classical Sobolev space equipped with the norm:

$$
\|v\|_{k, \Omega}:=\left(\sum_{0 \leq|\alpha| \leq k}\left\|D^{\alpha} v\right\|_{0, \Omega}^{2}\right)^{\frac{1}{2}}
$$

using the standard multi-index notation (the following convention is adopted: $H^{0}(\Omega):=L^{2}(\Omega),\|\quad\|_{0, \Omega}:=$ $\left.\|\quad\|_{L^{2}(\Omega)}\right)$. Fractional Sobolev spaces $H^{\tau}(\Omega), \tau \in \mathbb{R}^{+} \backslash \mathbb{N}$ are defined by

$$
H^{\tau}(\Omega)=\left\{v \in H^{k}(\Omega) \mid\|v\|_{\tau, \Omega}<+\infty\right\}
$$

where

$$
\|v\|_{\tau, \Omega}:=\left(\|v\|_{k, \Omega}^{2}+\sum_{|\alpha|=k} \int_{\Omega} \int_{\Omega} \frac{\left(D^{\alpha} v(\boldsymbol{x})-D^{\alpha} v(\boldsymbol{y})\right)^{2}}{|\boldsymbol{x}-\boldsymbol{y}|^{2+2 \theta}} \mathrm{d} \boldsymbol{x} \mathrm{d} \boldsymbol{y}\right)^{\frac{1}{2}}
$$

with $k$ being the integer part of $\tau$ and $\theta$ its decimal part (see $[1,11]$ ).

On any portion $\Gamma \subseteq \partial \Omega$ we introduce the space $H^{\frac{1}{2}}(\Gamma)$ as follows:

$$
H^{\frac{1}{2}}(\Gamma)=\left\{\psi \in L^{2}(\Gamma) \mid\|\psi\|_{\frac{1}{2}, \Gamma}<+\infty\right\},
$$

where

$$
\|\psi\|_{\frac{1}{2}, \Gamma}:=\left(\|\psi\|_{0, \Gamma}^{2}+\int_{\Gamma} \int_{\Gamma} \frac{(\psi(\boldsymbol{x})-\psi(\boldsymbol{y}))^{2}}{|\boldsymbol{x}-\boldsymbol{y}|^{2}} \mathrm{~d} \Gamma_{x} \mathrm{~d} \Gamma_{y}\right)^{\frac{1}{2}} .
$$

It is well-known that there exists a linear continuous mapping $E \in \mathscr{L}\left(H^{\frac{1}{2}}(\Gamma), H^{1}(\Omega)\right)$ such that

$$
E \psi_{\mid \Gamma}=\psi \quad \text { on } \Gamma
$$

where $E \psi_{\mid \Gamma}$ denotes the trace of $E \psi$ on $\Gamma$.

The topological dual space of $H^{\frac{1}{2}}(\Gamma)$ is denoted $H^{-\frac{1}{2}}(\Gamma),\langle,\rangle_{\frac{1}{2}, \Gamma}$ stands for the duality pairing and

$$
\|\mu\|_{-\frac{1}{2}, \Gamma}:=\sup _{\psi \in H^{\frac{1}{2}}(\Gamma), \gamma \neq 0} \frac{\langle\mu, \psi\rangle_{\frac{1}{2}, \Gamma}}{\|\psi\|_{\frac{1}{2}, \Gamma}}
$$

is the norm in $H^{-\frac{1}{2}}(\Gamma)$. 
The Cartesian product of $m$ previous spaces and their elements are denoted by bold characters. The respective norms are introduced as follows:

$$
\begin{aligned}
\|\boldsymbol{v}\|_{k, \Omega} & :=\left(\sum_{\ell=1}^{m}\left\|v_{\ell}\right\|_{k, \Omega}^{2}\right)^{1 / 2}, \boldsymbol{v}=\left(v_{1}, \cdots, v_{m}\right) \in \boldsymbol{H}^{k}(\Omega) \\
\|\boldsymbol{\psi}\|_{\frac{1}{2}, \Gamma}: & \left(\sum_{\ell=1}^{m}\left\|\psi_{\ell}\right\|_{\frac{1}{2}, \Gamma}^{2}\right)^{1 / 2}, \boldsymbol{\psi}=\left(\psi_{1}, \cdots, \psi_{m}\right) \in \boldsymbol{H}^{\frac{1}{2}}(\Gamma) \\
\|\boldsymbol{\mu}\|_{-\frac{1}{2}, \Gamma} & :=\left(\sum_{\ell=1}^{m}\left\|\mu_{\ell}\right\|_{-\frac{1}{2}, \Gamma}^{2}\right)^{1 / 2}, \boldsymbol{\mu}=\left(\mu_{1}, \cdots, \mu_{m}\right) \in \boldsymbol{H}^{-\frac{1}{2}}(\Gamma) .
\end{aligned}
$$

A special attention will be paid to the case when $k=1$ and $n=m=3$, i.e. $\Omega \subset \mathbb{R}^{3}$ and $\boldsymbol{H}^{1}(\Omega)=\left(H^{1}(\Omega)\right)^{3}$.

Let $\boldsymbol{V} \subset \boldsymbol{H}^{1}(\Omega)$ be a subspace of functions vanishing on a non-empty portion $\Gamma_{\boldsymbol{u}}$ open in $\partial \Omega$ :

$$
\boldsymbol{V}=\left\{\boldsymbol{v}=\left(v_{1}, v_{2}, v_{3}\right) \in \boldsymbol{H}^{1}(\Omega) \mid \quad v_{i}=0 \text { on } \Gamma_{\boldsymbol{u}}, i=1,2,3\right\}
$$

In addition to the classical norm \|\|$_{1, \Omega}$, we introduce the energetic norm \|\|$_{1, \Omega}$ in $\boldsymbol{V}$ corresponding to the scalar product

$$
((\boldsymbol{u}, \boldsymbol{v}))_{1, \Omega}:=\int_{\Omega} \sum_{i, j=1}^{3} \varepsilon_{i j}(\boldsymbol{u}) \varepsilon_{i j}(\boldsymbol{v}) \mathrm{d} \boldsymbol{x},
$$

where $\varepsilon_{i j}(\boldsymbol{u})$ is the $i, j$ th component of the linearized strain tensor

$$
\varepsilon(\boldsymbol{u}):=\frac{1}{2}\left(\nabla \boldsymbol{u}+(\nabla \boldsymbol{u})^{T}\right)
$$

From the Korn's inequality it follows that \|\|$_{1, \Omega}$ and $\|\mid\|_{1, \Omega}$ are equivalent in $\boldsymbol{V}$.

Let $\Gamma \subseteq \partial \Omega \backslash \bar{\Gamma}_{\boldsymbol{u}}$ be a portion of $\partial \Omega$ such that $\operatorname{dist}\left(\Gamma, \Gamma_{\boldsymbol{u}}\right)>0^{1}$ and denote by $\boldsymbol{H}_{\Gamma}^{\frac{1}{2}}$ the space of traces $\boldsymbol{v}_{\mid \Gamma}$ for $\boldsymbol{v} \in \boldsymbol{V}$. Besides the norm \|\|$_{\frac{1}{2}, \Gamma}$ defined by (2.1) we introduce the following quotient norm:

$$
\|\boldsymbol{\psi}\|_{\frac{1}{2}, \Gamma}:=\inf _{\boldsymbol{v} \in \boldsymbol{V}, \boldsymbol{v}=\boldsymbol{\psi} \text { on } \Gamma}\left\||\boldsymbol{v} \||_{1, \Omega} .\right.
$$

It is readily seen that

$$
\left.\left\|\left|\boldsymbol{\psi}\left\|_{\frac{1}{2}, \Gamma}=\right\|\right| \boldsymbol{w}_{\psi}\right\|\right|_{1, \Omega},
$$

where $\boldsymbol{w}_{\psi} \in \boldsymbol{V}$ is the unique solution of the elliptic problem:

$$
\left\{\begin{array}{l}
\left(\left(\boldsymbol{w}_{\psi}, \boldsymbol{v}\right)\right)_{1, \Omega}=0 \quad \forall \boldsymbol{v} \in \boldsymbol{V}_{0}=\{\boldsymbol{z} \in \boldsymbol{V} \mid \quad \boldsymbol{z}=\mathbf{0} \text { on } \Gamma\} \\
\boldsymbol{w}_{\psi}=\boldsymbol{\psi} \text { on } \Gamma
\end{array}\right.
$$

From the Banach theorem on the isomorphism it follows that \|\|$_{\frac{1}{2}, \Gamma}$ and $\left\||\||_{\frac{1}{2}, \Gamma}\right.$ are equivalent in $\boldsymbol{H}_{\Gamma}^{\frac{1}{2}}$.

Let $\boldsymbol{H}_{\Gamma}^{-\frac{1}{2}}$ be the dual space of $\boldsymbol{H}_{\Gamma}^{\frac{1}{2}}$ and denote \|\|$\|\left.\right|_{-\frac{1}{2}, \Gamma}$ the dual norm corresponding to \|\|$_{\frac{1}{2}, \Gamma}$ :

$$
\left\||\boldsymbol{\mu} \||_{-\frac{1}{2}, \Gamma}:=\sup _{\boldsymbol{\psi} \in \boldsymbol{H}_{\Gamma}^{\frac{1}{2}}, \boldsymbol{\psi} \neq \mathbf{0}} \frac{\langle\boldsymbol{\mu}, \boldsymbol{\psi}\rangle_{\frac{1}{2}, \Gamma}}{\|\mid \boldsymbol{\psi}\|_{\frac{1}{2}, \Gamma}}\right.
$$

\footnotetext{
${ }^{1}$ This assumption is not necessary but it simplifies the presentation.
} 
It is readily seen that

$$
\left.\left\|\left|\boldsymbol{\mu}\left\|\left.\right|_{-\frac{1}{2}, \Gamma}=\right\|\right| \boldsymbol{w}_{\mu}\right\|\right|_{1, \Omega}
$$

where $\boldsymbol{w}_{\mu} \in \boldsymbol{V}$ is the unique solution of the elliptic problem:

$$
\left(\left(\boldsymbol{w}_{\mu}, \boldsymbol{v}\right)\right)_{1, \Omega}=\langle\boldsymbol{\mu}, \boldsymbol{v}\rangle_{\frac{1}{2}, \Gamma} \quad \forall \boldsymbol{v} \in \boldsymbol{V} .
$$

In addition, \|\|$_{-\frac{1}{2}, \Gamma}$ and $\|\mid\|_{-\frac{1}{2}, \Gamma}$ are equivalent in $\boldsymbol{H}_{\Gamma}^{-\frac{1}{2}}$.

\section{Variational Formulation of the Signorini Problem With Given friction}

Let us consider an elastic body occupying a domain $\Omega \subset \mathbb{R}^{3}$ with the Lipschitz boundary $\partial \Omega$ which is split into three non-empty, non-overlapping parts $\Gamma_{\boldsymbol{u}}, \Gamma_{\boldsymbol{g}}$ and $\Gamma_{c}$, $\operatorname{dist}\left(\Gamma_{c}, \Gamma_{\boldsymbol{u}}\right)>0: \partial \Omega=\bar{\Gamma}_{\boldsymbol{u}} \cup \bar{\Gamma}_{\boldsymbol{g}} \cup \bar{\Gamma}_{c}$. For the sake of simplicity of our presentation we shall suppose that $\Omega=(0, a) \times(0, b) \times(0, c)$, where $a, b, c>0$ are given and $\Gamma_{c}=(0, a) \times(0, b) \times\{0\}$. The zero displacements are prescribed on $\Gamma_{\boldsymbol{u}}$ while surface tractions of density $\boldsymbol{g} \in \mathbf{L}^{2}\left(\Gamma_{\boldsymbol{g}}\right)$ act on $\Gamma_{\boldsymbol{g}}$. The body is unilaterally supported along $\Gamma_{c}$ by the rigid half-space

$$
S=\left\{\boldsymbol{x}=\left(x_{1}, x_{2}, x_{3}\right) \in \mathbb{R}^{3} \quad \mid x_{3} \leq 0\right\} .
$$

Besides constraints on the deformation of $\Omega$ we shall take into account effects of friction. We restrict ourselves to the simplest model, namely the model with given friction. Finally the body is subject to volume forces of density $\boldsymbol{f} \in \mathbf{L}^{2}(\Omega)$. Our aim is to find an equilibrium state of $\Omega$.

The classical formulation of the previous problem consists in finding a displacement vector $\boldsymbol{u}=\left(u_{1}, u_{2}, u_{3}\right)$ satisfying the equations and conditions (3.1)-(3.4):

$$
\begin{cases}\operatorname{div} \boldsymbol{\sigma}(\boldsymbol{u})+\boldsymbol{f} & =\mathbf{0} \text { in } \Omega \\ \boldsymbol{\sigma}(\boldsymbol{u}) \boldsymbol{n}-\boldsymbol{g} & =\mathbf{0} \text { on } \Gamma_{\boldsymbol{g}} \\ \boldsymbol{u} & =\mathbf{0} \text { on } \Gamma_{\boldsymbol{u}}\end{cases}
$$

The symbol $\boldsymbol{\sigma}(\boldsymbol{u})=\left(\sigma_{i j}(\boldsymbol{u})\right)_{i, j=1}^{3}$ stands for a symmetric stress tensor which is related to the linearized strain tensor $\varepsilon(\boldsymbol{u})$ by means of a linear Hooke's law:

$$
\boldsymbol{\sigma}(\boldsymbol{u})=A \varepsilon(\boldsymbol{u}),
$$

where $A$ is a fourth order symmetric tensor satisfying the usual ellipticity conditions in $\Omega$. Further $\boldsymbol{n}$ is the outward unit normal vector to $\partial \Omega$ and div denotes the divergence operator acting on tensor functions (the summation convention is adopted):

$$
\operatorname{div} \boldsymbol{\sigma}=\left(\frac{\partial \sigma_{i j}}{\partial x_{j}}\right)_{i=1}^{3} .
$$

Let $\boldsymbol{u}_{n}:=u_{n} \boldsymbol{n}, \boldsymbol{u}_{t}:=\boldsymbol{u}-u_{n} \boldsymbol{n}$ be the normal, tangential component of the displacement vector $\boldsymbol{u}$ and $\boldsymbol{\sigma}_{n}(\boldsymbol{u}):=\sigma_{n}(\boldsymbol{u}) \boldsymbol{n}$, and $\boldsymbol{\sigma}_{t}\left(\boldsymbol{u}_{t}\right):=\boldsymbol{\sigma}(\boldsymbol{u}) \boldsymbol{n}-\sigma_{n}(\boldsymbol{u}) \boldsymbol{n}$ the normal, tangential component of the stress vector $\boldsymbol{\sigma}(\boldsymbol{u}) \boldsymbol{n}$, respectively, where $u_{n}:=\boldsymbol{u} \cdot \boldsymbol{n}$ and $\sigma_{n}(\boldsymbol{u}):=(\boldsymbol{\sigma}(\boldsymbol{u}) \boldsymbol{n}) \cdot \boldsymbol{n}$. Here $\cdot$ denotes the scalar product of two vectors.

The unilateral and friction conditions prescribed on $\Gamma_{c}$ read as follows:

$$
\begin{gathered}
u_{n} \leq 0, \quad \sigma_{n}(\boldsymbol{u}) \leq 0, \quad u_{n} \sigma_{n}(\boldsymbol{u})=0 \quad \text { on } \Gamma_{c}, \\
\left\{\begin{array}{l}
\left\|\boldsymbol{\sigma}_{t}(\boldsymbol{u})\right\| \leq s \quad \text { a.e. on } \Gamma_{c}, \\
\text { if }\left\|\boldsymbol{\sigma}_{t}(\boldsymbol{u})(\boldsymbol{x})\right\|<s(\boldsymbol{x}) \text { then } \boldsymbol{u}_{t}(\boldsymbol{x})=\mathbf{0}, \quad \boldsymbol{x} \in \Gamma_{c}, \\
\text { if }\left\|\boldsymbol{\sigma}_{t}(\boldsymbol{u})(\boldsymbol{x})\right\|=s(\boldsymbol{x})>0 \text { then there exists } \nu(\boldsymbol{x}) \geq 0 \\
\text { such that } \boldsymbol{u}_{t}(\boldsymbol{x})=-\nu(\boldsymbol{x}) \boldsymbol{\sigma}_{t}(\boldsymbol{u}(\boldsymbol{x})), \quad \boldsymbol{x} \in \Gamma_{c} .
\end{array}\right.
\end{gathered}
$$

Here $s \in L^{2}\left(\Gamma_{c}\right), s \geq 0$ is a given function and $\left\|\boldsymbol{\sigma}_{t}(\boldsymbol{u})\right\|$ is the Euclidean norm of $\boldsymbol{\sigma}_{t}(\boldsymbol{u})$. 
Remark 3.1. In the classical Coulomb's law of friction $[2,6]$ the given slip bound $s$ is replaced by the term $\mathbf{F}\left|\sigma_{n}(\boldsymbol{u})\right|$ which is not known a priori. The symbol $\mathbf{F}$ stands for the coefficient of Coulomb friction.

Remark 3.2. Taking into account the fact that $\boldsymbol{n}=(0,0,-1)$ on $\Gamma_{c}$, we have:

$$
\begin{gathered}
u_{n}=-u_{3}, \sigma_{n}(\boldsymbol{u})=\sigma_{33}(\boldsymbol{u}), \\
\boldsymbol{\sigma}_{t}(\boldsymbol{u})=\left(-\sigma_{13}(\boldsymbol{u}),-\sigma_{23}(\boldsymbol{u}), 0\right) .
\end{gathered}
$$

Remark 3.3. Let $\boldsymbol{t}_{1}(\boldsymbol{x}), \boldsymbol{t}_{2}(\boldsymbol{x}), \boldsymbol{x} \in \Gamma_{c}$ be two unit vectors such that the triplet $\left\{\boldsymbol{n}(\boldsymbol{x}), \boldsymbol{t}_{1}(\boldsymbol{x}), \boldsymbol{t}_{2}(\boldsymbol{x})\right\}$ forms a local orthonormal basis in $\mathbb{R}^{3}$ with origin at $\boldsymbol{x} \in \Gamma_{c}$. Then any vector function $\boldsymbol{w}: \Gamma_{c} \longrightarrow \mathbb{R}^{3}$ can be represented in the local coordinate system $\left\{\boldsymbol{n}(\boldsymbol{x}), \boldsymbol{t}_{1}(\boldsymbol{x}), \boldsymbol{t}_{2}(\boldsymbol{x})\right\}$ as follows:

$$
\boldsymbol{w}(\boldsymbol{x})=\left(w_{n}(\boldsymbol{x}), \boldsymbol{w}_{t}(\boldsymbol{x})\right) \in \mathbb{R} \times \mathbb{R}^{2},
$$

where $w_{n}(\boldsymbol{x})=\boldsymbol{w}(\boldsymbol{x}) \cdot \boldsymbol{n}, \boldsymbol{w}_{t}(\boldsymbol{x})=\left(w_{t_{1}}(\boldsymbol{x}), w_{t_{2}}(\boldsymbol{x})\right), w_{t_{j}}(\boldsymbol{x})=\boldsymbol{w}(\boldsymbol{x}) \cdot \boldsymbol{t}_{j}(\boldsymbol{x}), j=1,2$. In particular, we shall use this representation for the trace of displacement vectors $\boldsymbol{v}$ on $\Gamma_{c}$ in what follows, i.e. $\boldsymbol{v}(\boldsymbol{x})=\left(v_{n}(\boldsymbol{x}), \boldsymbol{v}_{t}(\boldsymbol{x})\right) \in$ $\mathbb{R} \times \mathbb{R}^{2}, \boldsymbol{x} \in \Gamma_{c}$ and

$$
\left\|\boldsymbol{v}_{t}\right\|=\left(\left|v_{t_{1}}\right|^{2}+\left|v_{t_{2}}\right|^{2}\right)^{\frac{1}{2}} \quad \text { a.e. on } \Gamma_{c} .
$$

To give the variational formulation of our problem we first introduce the Hilbert space $\boldsymbol{V}$ :

$$
\boldsymbol{V}=\left\{\boldsymbol{v} \in \boldsymbol{H}^{1}(\Omega) \mid \boldsymbol{v}=\mathbf{0} \text { on } \Gamma_{\boldsymbol{u}}\right\},
$$

and its closed convex subset $\boldsymbol{K}$ of kinematically admissible displacements:

$$
\boldsymbol{K}=\left\{\boldsymbol{v} \in \boldsymbol{V} \mid \quad v_{n} \leq 0 \text { on } \Gamma_{c}\right\} .
$$

Next, we define:

$$
\begin{gathered}
a(\boldsymbol{u}, \boldsymbol{v}):=\int_{\Omega} A \boldsymbol{\varepsilon}(\boldsymbol{u}) \boldsymbol{\varepsilon}(\boldsymbol{v}) \mathrm{d} \boldsymbol{x}, \\
L(\boldsymbol{v}):=\int_{\Omega} \boldsymbol{f} \cdot \boldsymbol{v} \mathrm{d} \boldsymbol{x}+\int_{\Gamma_{\boldsymbol{g}}} \boldsymbol{g} \cdot \boldsymbol{v} \mathrm{d} \Gamma \\
j(\boldsymbol{v}):=\int_{\Gamma_{c}} s\left\|\boldsymbol{v}_{t}\right\| \mathrm{d} \Gamma .
\end{gathered}
$$

The primal variational formulation of the contact problem with given friction reads as follows:

$$
\left\{\begin{array}{l}
\text { Find } \boldsymbol{u} \in \boldsymbol{K} \text { such that } \\
\mathbf{J}(\boldsymbol{u}) \leq \mathbf{J}(\boldsymbol{v}) \quad \forall \boldsymbol{v} \in \boldsymbol{K}
\end{array}\right.
$$

where

is the total potential energy functional.

$$
\mathbf{J}(\boldsymbol{v})=\frac{1}{2} a(\boldsymbol{v}, \boldsymbol{v})-L(\boldsymbol{v})+j(\boldsymbol{v})
$$


On the basis of the previous assumptions we have:

Theorem 3.1. Problem (3.5) has a unique solution.

In order to release the unilateral constraint $v_{n} \leq 0$ on $\Gamma_{c}$ and to regularize the non-differentiable term $j$ we use a duality approach. To this end we introduce several notations. Let $\gamma_{n}: \boldsymbol{V} \longrightarrow L^{2}\left(\Gamma_{c}\right)$ be the trace mapping defined by

Further let

$$
\gamma_{n} \boldsymbol{v}:=v_{n} \text { on } \Gamma_{c}, \boldsymbol{v} \in \boldsymbol{V}
$$

$$
\begin{gathered}
H_{\Gamma_{c}}^{\frac{1}{2}}:=\gamma_{n} \boldsymbol{V}, H_{\Gamma_{c}}^{-\frac{1}{2}}:=\text { the dual of } H_{\Gamma_{c}}^{\frac{1}{2}}, \\
\boldsymbol{H}_{\Gamma_{c}}^{\frac{1}{2}}:=\left(H_{\Gamma_{c}}^{\frac{1}{2}}\right)^{3}, \quad \boldsymbol{H}_{\Gamma_{c}}^{-\frac{1}{2}}:=\left(H_{\Gamma_{c}}^{-\frac{1}{2}}\right)^{3}, \\
M_{n}=\left\{\mu_{n} \in H_{\Gamma_{c}}^{-\frac{1}{2}}, \quad \mu_{n} \geq 0 \text { on } \Gamma_{c}\right\}, \\
\mathbf{M}_{t}=\left\{\boldsymbol{\mu}_{t}=\left(\mu_{t_{1}}, \mu_{t_{2}}\right) \in \mathbf{L}^{2}\left(\Gamma_{c}\right), \quad\left\|\boldsymbol{\mu}_{t}\right\| \leq s \quad \text { a.e. on } \Gamma_{c}\right\} \\
=\left\{\boldsymbol{\mu}_{t} \in \mathbf{L}^{2}\left(\Gamma_{c}\right) \mid \int_{\Gamma_{c}} \boldsymbol{\mu}_{t} \cdot \boldsymbol{\psi} d \Gamma-\int_{\Gamma_{c}} s\|\boldsymbol{\psi}\| d \Gamma \leq 0 \quad \forall \boldsymbol{\psi} \in \mathbf{L}^{2}\left(\Gamma_{c}\right)\right\} .
\end{gathered}
$$

Remark 3.4. In accordance with the previous section, the spaces $\boldsymbol{H}_{\Gamma_{c}}^{\frac{1}{2}}, \boldsymbol{H}_{\Gamma_{c}}^{-\frac{1}{2}}$ will be endowed with two equivalent norms \|\|$_{\frac{1}{2}, \Gamma_{c}},\|\| \|_{\frac{1}{2}, \Gamma_{c}}$ and \|\|$_{-\frac{1}{2}, \Gamma_{c}},\|\| \quad \|\left.\right|_{-\frac{1}{2}, \Gamma_{c}}$, respectively.

It is easy to verify that

$$
\min _{\boldsymbol{K}} \mathbf{J}(\boldsymbol{v})=\min _{\boldsymbol{V}} \sup _{M_{n} \times \mathbf{M}_{t}} \mathbf{L}\left(\boldsymbol{v}, \mu_{n}, \boldsymbol{\mu}_{t}\right)
$$

where $\mathbf{L}: \boldsymbol{V} \times M_{n} \times \mathbf{M}_{t} \longrightarrow \mathbb{R}$ is the Lagrangian defined by

$$
\mathbf{L}\left(\boldsymbol{v}, \mu_{n}, \boldsymbol{\mu}_{t}\right)=\mathbf{J}(\boldsymbol{v})+\left\langle\mu_{n}, v_{n}\right\rangle_{\frac{1}{2}, \Gamma_{c}}+\int_{\Gamma_{c}} \boldsymbol{\mu}_{t} \cdot \boldsymbol{v}_{t} \mathrm{~d} \Gamma
$$

and $\langle., .\rangle_{\frac{1}{2}, \Gamma_{c}}$ stands for the duality pairing between $H_{\Gamma_{c}}^{-\frac{1}{2}}$ and $H_{\Gamma_{c}}^{\frac{1}{2}}$.

By a mixed formulation of (3.5) we call a problem of finding a saddle-point of $\mathbf{L}$ on $\boldsymbol{V} \times M_{n} \times \mathbf{M}_{t}$ :

$$
\left\{\begin{array}{c}
\text { Find }\left(\boldsymbol{w}, \lambda_{n}, \boldsymbol{\lambda}_{t}\right) \in \boldsymbol{V} \times M_{n} \times \mathbf{M}_{t} \text { such that } \\
\mathbf{L}\left(\boldsymbol{w}, \mu_{n}, \boldsymbol{\mu}_{t}\right) \leq \mathbf{L}\left(\boldsymbol{w}, \lambda_{n}, \boldsymbol{\lambda}_{t}\right) \leq \mathbf{L}\left(\boldsymbol{v}, \lambda_{n}, \boldsymbol{\lambda}_{t}\right) \quad \forall\left(\boldsymbol{v}, \mu_{n}, \boldsymbol{\mu}_{t}\right) \in \boldsymbol{V} \times M_{n} \times \mathbf{M}_{t},
\end{array}\right.
$$

or equivalently

$$
\left\{\begin{array}{c}
\text { Find }(\boldsymbol{w}, \boldsymbol{\lambda}) \in \boldsymbol{V} \times \mathbf{M} \text { such that } \\
a(\boldsymbol{w}, \boldsymbol{v})+b(\boldsymbol{\lambda}, \boldsymbol{v})=L(\boldsymbol{v}), \quad \forall \boldsymbol{v} \in \boldsymbol{V} \\
b(\boldsymbol{\mu}-\boldsymbol{\lambda}, \boldsymbol{w}) \leq 0, \quad \forall \boldsymbol{\mu} \in \mathbf{M}
\end{array}\right.
$$

where $\boldsymbol{\lambda}:=\left(\lambda_{n}, \boldsymbol{\lambda}_{t}\right), \mathbf{M}:=M_{n} \times \mathbf{M}_{t}$ and

$$
b(\boldsymbol{\mu}, \boldsymbol{v}):=\left\langle\mu_{n}, v_{n}\right\rangle_{\frac{1}{2}, \Gamma_{c}}+\int_{\Gamma_{c}} \boldsymbol{\mu}_{t} \cdot \boldsymbol{v}_{t} \mathrm{~d} \Gamma, \quad \boldsymbol{\mu} \in \mathbf{M}, \boldsymbol{v} \in \boldsymbol{V}
$$

The relation between (3.5) and (3.6) follows from the next theorem. 
Theorem 3.2. There exists a unique solution $(\boldsymbol{w}, \boldsymbol{\lambda})$ of (3.6). In addition,

$$
\boldsymbol{w}=\boldsymbol{u} \quad \text { in } \Omega, \quad \lambda_{n}=-\sigma_{n}(\boldsymbol{u}), \quad \boldsymbol{\lambda}_{t}=-\boldsymbol{\sigma}_{t}(\boldsymbol{u}) \quad \text { on } \Gamma_{c},
$$

where $\boldsymbol{u} \in \boldsymbol{K}$ is the solution to (3.5).

Proof is obvious.

Convention: the solution to (3.6) will be denoted by $\left(\boldsymbol{u}, \lambda_{n}, \boldsymbol{\lambda}_{t}\right)$ in what follows.

\section{Finite element approximation}

The present section is devoted to a finite element approximation of the saddle-point problem (3.6). The key point lies in the finite element discretization of the closed convex set $\mathbf{M}$ of Lagrange multipliers which leads to a well-posed discrete problem and gives also a good convergence rate for approximate solutions.

We start with discretizations of $\boldsymbol{V}, M_{n}$ and $\mathbf{M}_{t}$.

Let $\left\{\mathbf{T}_{h}\right\}, h \rightarrow 0+$ be a regular family (see [5]) of partitions of $\bar{\Omega}$ into tetrahedrons $\kappa$. With any $\mathbf{T}_{h} \in\left\{\mathbf{T}_{h}\right\}$ we associate the following space of continuous piecewise linear functions:

$$
\begin{gathered}
V_{h}=\left\{v_{h} \in \mathbf{C}(\bar{\Omega}) \mid \quad v_{h \mid \kappa} \in \mathbb{P}_{1}(\kappa) \quad \forall \kappa \in \mathbf{T}_{h}, \quad v_{h}=0 \quad \text { on } \Gamma_{\boldsymbol{u}}\right\}, \\
\boldsymbol{V}_{h}=\left(V_{h}\right)^{3} .
\end{gathered}
$$

Further, let $\left\{\mathcal{R}_{H}\right\}, H \rightarrow 0+$ be a strongly regular family of rectangulations of $\bar{\Gamma}_{c}$ with $H>0$ being the norm of $\mathcal{R}_{H}$. On every $\mathcal{R}_{H} \in\left\{\mathcal{R}_{H}\right\}$ we construct the space of piecewise constant functions:

$$
L_{H}=\left\{\mu_{H} \in L^{2}\left(\Gamma_{c}\right) \mid \quad \mu_{H \mid \mathcal{R}} \in \mathbb{P}_{0}(\mathcal{R}) \forall \mathcal{R} \in \mathcal{R}_{H}\right\}
$$

$$
\mathbf{L}_{H}=\left(L_{H}\right)^{3}
$$

and the following convex sets:

$$
\begin{gathered}
M_{H n}=\left\{\mu_{H n} \in L_{H} \mid \quad \mu_{H n} \geq 0 \quad \text { a.e. on } \Gamma_{c}\right\} \\
\mathbf{M}_{H t}=\left\{\boldsymbol{\mu}_{H t} \in\left(L_{H}\right)^{2} \mid \quad \int_{\Gamma_{c}} \boldsymbol{\mu}_{H t} \cdot \boldsymbol{\psi}_{H} d \Gamma-\int_{\Gamma_{c}} s\left\|\boldsymbol{\psi}_{H}\right\| d \Gamma \leq 0 \forall \boldsymbol{\psi}_{H} \in\left(L_{H}\right)^{2}\right\} .
\end{gathered}
$$

Recall that $\|\cdot\|$ stands for the Euclidean norm in $\mathbb{R}^{2}$. The set $\mathbf{M}_{H t}$ is the external approximation of $\mathbf{M}_{t}$, while $M_{H n}$ is the internal approximation of $M_{n}$.

The discretization of (3.6) is defined in a standard way:

$$
\left\{\begin{array}{r}
\text { Find } \boldsymbol{u}_{h} \in \boldsymbol{V}_{h} \text { and } \boldsymbol{\lambda}_{H} \in \mathbf{M}_{H} \text { such that } \\
a\left(\boldsymbol{u}_{h}, \boldsymbol{v}_{h}\right)+b\left(\boldsymbol{\lambda}_{H}, \boldsymbol{v}_{h}\right)=L\left(\boldsymbol{v}_{h}\right), \quad \forall \boldsymbol{v}_{h} \in \boldsymbol{V}_{h}, \\
b\left(\boldsymbol{\mu}_{H}-\boldsymbol{\lambda}_{H}, \boldsymbol{u}_{h}\right) \leq 0, \quad \forall \boldsymbol{\mu}_{H} \in \mathbf{M}_{H},
\end{array}\right.
$$

where $\boldsymbol{\lambda}_{H}:=\left(\lambda_{H n}, \boldsymbol{\lambda}_{H t}\right)$ and $\mathbf{M}_{H}:=M_{H n} \times \mathbf{M}_{H t}$. To ensure the existence and uniqueness of a solution to (4.3), the following stability condition is needed:

$$
\left\{\boldsymbol{\mu}_{H} \in \mathbf{L}_{H} \mid \quad b\left(\boldsymbol{\mu}_{H}, \boldsymbol{v}_{h}\right)=0 \forall \boldsymbol{v}_{h} \in \boldsymbol{V}_{h}\right\}=\{\mathbf{0}\} .
$$


In the forthcoming convergence analysis, we will need more information on the compatibility between the spaces $\mathbf{L}_{H}$ and $\boldsymbol{V}_{h}$. We shall introduce a stronger assumption, namely the Ladyzhenskaya-Babuska-Brezzi condition which in our particular case takes the form

$$
\sup _{\boldsymbol{v}_{h} \in \boldsymbol{V}_{h}} \frac{b\left(\boldsymbol{\mu}_{H}, \boldsymbol{v}_{h}\right)}{\left.\left\|\boldsymbol{v}_{h}\right\|\right|_{1, \Omega}} \geq \beta\left\|\boldsymbol{\mu}_{H}\right\|_{-\frac{1}{2}, \Gamma_{c}} \quad \forall \boldsymbol{\mu}_{H} \in \mathbf{L}_{H},
$$

where $\beta>0$ is independent of $h$ and $H$. The dual norm $\left\||\||_{-\frac{1}{2}, \Gamma_{c}}\right.$ in (4.5) is evaluated by means of (2.3). Next we shall suppose that the auxiliary problem (2.4) is regular in the following sense:

$$
\left\{\begin{array}{l}
\text { there exists } \varepsilon \in(0,1 / 2) \text { such that for every } \boldsymbol{\mu} \in \boldsymbol{H}_{\Gamma_{c}}^{-\frac{1}{2}+\varepsilon}:=\text { the dual of } \boldsymbol{H}_{\Gamma_{c}}^{\frac{1}{2}-\varepsilon} \\
\text { the solution } \boldsymbol{w}_{\mu} \text { of }(2.4) \text { belongs to } \boldsymbol{V} \cap \boldsymbol{H}^{1+\varepsilon}(\Omega) \text { and } \\
\left\|\boldsymbol{w}_{\mu}\right\|_{1+\varepsilon, \Omega} \leq C(\varepsilon)\|\boldsymbol{\mu}\|_{-\frac{1}{2}+\varepsilon, \Gamma_{c}}
\end{array}\right.
$$

holds with a positive constant $C(\varepsilon)$ which depends solely on $\varepsilon>0$. If it is so then using exactly the same approach as in [12] one can prove the next lemma.

Lemma 4.1. Let us suppose that (2.4) is regular, $\left\{\mathcal{R}_{H}\right\}$ is a strongly regular family of rectangulations of $\bar{\Gamma}_{c}$ and the ratio $h / H$ is sufficiently small. Then the Ladyzhenskaya-Babuska-Brezzi condition (4.5) is satisfied.

\section{ERROR ANALYSIS}

In this section we establish a priori error estimates for the mixed finite element approximation (4.3). We start with the following lemma [3]:

Lemma 5.1. Let $(\boldsymbol{u}, \boldsymbol{\lambda}),\left(\boldsymbol{u}_{h}, \boldsymbol{\lambda}_{H}\right)$ be the solution of (3.6), (4.3), respectively. Then for any $\boldsymbol{v}_{h} \in \boldsymbol{V}_{h}$ and $\boldsymbol{\mu}_{H} \in \mathbf{M}_{H}$ it holds:

$$
\begin{aligned}
a\left(\boldsymbol{u}-\boldsymbol{u}_{h}, \boldsymbol{u}-\boldsymbol{u}_{h}\right) \leq & a\left(\boldsymbol{u}-\boldsymbol{u}_{h}, \boldsymbol{u}-\boldsymbol{v}_{h}\right)+b\left(\boldsymbol{\lambda}-\boldsymbol{\mu}_{H}, \boldsymbol{u}_{h}-\boldsymbol{u}\right)+b\left(\boldsymbol{\lambda}-\boldsymbol{\lambda}_{H}, \boldsymbol{u}-\boldsymbol{v}_{h}\right) \\
& +b\left(\boldsymbol{\lambda}-\boldsymbol{\mu}_{H}, \boldsymbol{u}\right)+b\left(\boldsymbol{\lambda}_{H}-\boldsymbol{\lambda}, \boldsymbol{u}\right) .
\end{aligned}
$$

Proof. Let $\boldsymbol{v}_{h}$ be an element of $\boldsymbol{V}_{h}$. Then

$$
a\left(\boldsymbol{u}-\boldsymbol{u}_{h}, \boldsymbol{u}-\boldsymbol{u}_{h}\right)=a\left(\boldsymbol{u}-\boldsymbol{u}_{h}, \boldsymbol{u}-\boldsymbol{v}_{h}\right)+a\left(\boldsymbol{u}-\boldsymbol{u}_{h}, \boldsymbol{v}_{h}-\boldsymbol{u}_{h}\right) .
$$

From the equations in (3.6) and (4.3), we obtain:

$$
\begin{aligned}
a\left(\boldsymbol{u}-\boldsymbol{u}_{h}, \boldsymbol{v}_{h}-\boldsymbol{u}_{h}\right) & =L\left(\boldsymbol{v}_{h}-\boldsymbol{u}_{h}\right)-b\left(\boldsymbol{\lambda}, \boldsymbol{v}_{h}-\boldsymbol{u}_{h}\right)-L\left(\boldsymbol{v}_{h}-\boldsymbol{u}_{h}\right)+b\left(\boldsymbol{\lambda}_{H}, \boldsymbol{v}_{h}-\boldsymbol{u}_{h}\right) \\
& =b\left(\boldsymbol{\lambda}, \boldsymbol{u}_{h}-\boldsymbol{v}_{h}\right)+b\left(\boldsymbol{\lambda}_{H}, \boldsymbol{v}_{h}-\boldsymbol{u}_{h}\right) .
\end{aligned}
$$

Further

and finally

$$
a\left(\boldsymbol{u}-\boldsymbol{u}_{h}, \boldsymbol{u}-\boldsymbol{u}_{h}\right)=a\left(\boldsymbol{u}-\boldsymbol{u}_{h}, \boldsymbol{u}-\boldsymbol{v}_{h}\right)+b\left(\boldsymbol{\lambda}, \boldsymbol{u}_{h}-\boldsymbol{v}_{h}\right)+b\left(\boldsymbol{\lambda}_{H}, \boldsymbol{v}_{h}-\boldsymbol{u}_{h}\right),
$$

$$
\begin{aligned}
\boldsymbol{a}\left(\boldsymbol{u}-\boldsymbol{u}_{h}, \boldsymbol{u}-\boldsymbol{u}_{h}\right)= & a\left(\boldsymbol{u}-\boldsymbol{u}_{h}, \boldsymbol{u}-\boldsymbol{v}_{h}\right)+b\left(\boldsymbol{\mu}_{H}-\boldsymbol{\lambda}, \boldsymbol{u}-\boldsymbol{u}_{h}\right)+b\left(\boldsymbol{\lambda}-\boldsymbol{\lambda}_{H}, \boldsymbol{u}-\boldsymbol{v}_{h}\right) \\
& +b\left(\boldsymbol{\lambda}-\boldsymbol{\mu}_{H}, \boldsymbol{u}\right)+b\left(\boldsymbol{\lambda}_{H}-\boldsymbol{\lambda}, \boldsymbol{u}\right)+b\left(\boldsymbol{\mu}_{H}-\boldsymbol{\lambda}_{H}, \boldsymbol{u}_{h}\right) .
\end{aligned}
$$

The inequality in (4.3) implies that $b\left(\boldsymbol{\mu}_{H}-\boldsymbol{\lambda}_{H}, \boldsymbol{u}_{h}\right) \leq 0$ for any $\boldsymbol{\mu}_{H} \in \mathbf{M}_{H}$. From this the error estimate (5.1) follows. 
We now derive an upper bound for $\left\|\boldsymbol{u}-\boldsymbol{u}_{h}\right\|_{1, \Omega}$.

Lemma 5.2. Let $(\boldsymbol{u}, \boldsymbol{\lambda}),\left(\boldsymbol{u}_{h}, \boldsymbol{\lambda}_{H}\right)$ be the solution of (3.6), (4.3), respectively. Suppose that $\boldsymbol{u} \in \boldsymbol{H}^{2}(\Omega)$ and the fourth order tensor $A$ defining the Hooke's law is sufficiently smooth. Then

$$
\left\|\boldsymbol{u}-\boldsymbol{u}_{h}\right\|_{1, \Omega}^{2} \leq C\left[h\left\|\boldsymbol{\lambda}-\boldsymbol{\lambda}_{H}\right\|_{-\frac{1}{2}, \Gamma_{c}}+H^{\frac{1}{2}+\varepsilon}\left\|\boldsymbol{\lambda}-\boldsymbol{\lambda}_{H}\right\|_{-\frac{1}{2}+\varepsilon, \Gamma_{c}}+H^{\frac{3}{2}}+H\right]
$$

where $\varepsilon>0$ is arbitrarily small and $C>0$ is a positive constant which does not depend on $h, H$.

Proof. We shall estimate each term on the right hand side of (5.1). But before proving these estimates, let us recall some approximation results. Let $\mathbf{I}_{h}$ be the Lagrange interpolation operator with values in $\boldsymbol{V}_{h}$. There exists a constant $C>0$ which does not depend on $h$ and such that for all $\boldsymbol{v} \in \boldsymbol{H}^{2}(\Omega)$ (see [5]) it holds:

$$
\left\|\boldsymbol{v}-\mathbf{I}_{h} \boldsymbol{v}\right\|_{1, \Omega} \leq C h\|\boldsymbol{v}\|_{2, \Omega}
$$

Next, we introduce the projection operator $\boldsymbol{\Pi}_{H}: \mathbf{L}^{2}\left(\Gamma_{c}\right) \longrightarrow \mathbf{L}_{H}$ by

$$
\boldsymbol{\Pi}_{H} \boldsymbol{\varphi} \in \mathbf{L}_{H}, \quad \int_{\Gamma_{c}}\left(\boldsymbol{\Pi}_{H} \boldsymbol{\varphi}-\boldsymbol{\varphi}\right) \cdot \boldsymbol{\mu}_{H} \mathrm{~d} \Gamma=0, \quad \forall \boldsymbol{\mu}_{H} \in \mathbf{L}_{H}
$$

The operator $\boldsymbol{\Pi}_{H}$ has the following approximation property (see [19]): there exists a positive constant $C$ independent of $H$ such that $\forall \boldsymbol{\varphi} \in \boldsymbol{H}_{\Gamma_{c}}^{\nu}, \nu=\frac{1}{2}, 1$ and any $\mu \in\left[0, \frac{1}{2}\right)$, it holds that

$$
\left\|\boldsymbol{\varphi}-\Pi_{H} \boldsymbol{\varphi}\right\|_{\mu, \Gamma_{c}} \leq C H^{\nu-\mu}\|\varphi\|_{\nu, \Gamma_{c}}
$$

Moreover, if $\varphi \in \mathbf{L}^{2}\left(\Gamma_{c}\right)$ then

$$
\left\|\boldsymbol{\varphi}-\boldsymbol{\Pi}_{H} \boldsymbol{\varphi}\right\|_{-\frac{1}{2}+\varepsilon, \Gamma_{c}} \leq C H^{\frac{1}{2}-\varepsilon}\left\|\boldsymbol{\varphi}-\boldsymbol{\Pi}_{H} \boldsymbol{\varphi}\right\|_{0, \Gamma_{c}}, \varepsilon \in\left[0, \frac{1}{2}\right) .
$$

Finally, let us note that the trace theorem implies that

$$
\|\boldsymbol{\lambda}\|_{\frac{1}{2}, \Gamma_{c}} \leq C\|\boldsymbol{u}\|_{2, \Omega}
$$

provided that the tensor $A$ is sufficiently smooth (the constant $C>0$ depends only on $A$ ).

Next we estimate each term appearing on the right hand side of (5.1) by choosing: $\boldsymbol{v}_{h}=\mathbf{I}_{h} \boldsymbol{u}$ and $\boldsymbol{\mu}_{H}=$ $\boldsymbol{\Pi}_{H} \boldsymbol{\lambda}=\left(\pi_{H n} \lambda_{n}, \boldsymbol{\pi}_{H t} \boldsymbol{\lambda}_{t}\right)$, where $\pi_{H n}$ and $\boldsymbol{\pi}_{H t}$ are the components of $\boldsymbol{\Pi}_{H}$ in $L_{H}$ and $\left(L_{H}\right)^{2}$, respectively. It is readily seen that $\boldsymbol{\Pi}_{H} \boldsymbol{\lambda} \in \mathbf{M}_{H}$ as follows from the definition of $\mathbf{M}, \mathbf{M}_{H}$ and (5.4).

(i) The first term is evaluated by using continuity of $a(.,$.$) and (5.3):$

$$
a\left(\boldsymbol{u}-\boldsymbol{u}_{h}, \boldsymbol{u}-\boldsymbol{v}_{h}\right) \leq C h\left\|\boldsymbol{u}-\boldsymbol{u}_{h}\right\|_{1, \Omega}
$$

(ii) The second is estimated by using (5.5), (5.6), (5.7) and the trace theorem:

$$
b\left(\boldsymbol{\lambda}-\boldsymbol{\mu}_{H}, \boldsymbol{u}_{h}-\boldsymbol{u}\right) \leq C\left\|\boldsymbol{\lambda}-\boldsymbol{\Pi}_{H} \boldsymbol{\lambda}\right\|_{-\frac{1}{2}, \Gamma_{c}}\left\|\boldsymbol{u}_{h}-\boldsymbol{u}\right\|_{1, \Omega} \leq C H\left\|\boldsymbol{u}_{h}-\boldsymbol{u}\right\|_{1, \Omega} .
$$

(iii) The third term is estimated as follows:

$$
b\left(\boldsymbol{\lambda}-\boldsymbol{\lambda}_{H}, \boldsymbol{u}-\boldsymbol{v}_{h}\right) \leq C\left\|\boldsymbol{\lambda}-\boldsymbol{\lambda}_{H}\right\|_{-\frac{1}{2}, \Gamma_{c}}\left\|\boldsymbol{u}-\boldsymbol{v}_{h}\right\|_{1, \Omega} \leq C h\left\|\boldsymbol{\lambda}-\boldsymbol{\lambda}_{H}\right\|_{-\frac{1}{2}, \Gamma_{c}} .
$$


(iv) To evaluate the fourth term, we invoke the definition of the $L^{2}$-projection operators:

$$
\begin{aligned}
b\left(\boldsymbol{\lambda}-\boldsymbol{\mu}_{H}, \boldsymbol{u}\right)= & \int_{\Gamma_{c}}\left(\lambda_{n}-\pi_{H n} \lambda_{n}\right) u_{n} \mathrm{~d} \Gamma+\int_{\Gamma_{c}}\left(\boldsymbol{\lambda}_{t}-\boldsymbol{\pi}_{H t} \boldsymbol{\lambda}_{t}\right) \cdot \boldsymbol{u}_{t} \mathrm{~d} \Gamma \\
= & \int_{\Gamma_{c}}\left(\lambda_{n}-\pi_{H n} \lambda_{n}\right)\left(u_{n}-\pi_{H n} u_{n}\right) \mathrm{d} \Gamma+\int_{\Gamma_{c}}\left(\boldsymbol{\lambda}_{t}-\boldsymbol{\pi}_{H t} \boldsymbol{\lambda}_{t}\right) \cdot\left(\boldsymbol{u}_{t}-\boldsymbol{\pi}_{H t} \boldsymbol{u}_{t}\right) \mathrm{d} \Gamma \\
\leq & \left\|\lambda_{n}-\pi_{H n} \lambda_{n}\right\|_{0, \Gamma_{c}}\left\|u_{n}-\pi_{H n} u_{n}\right\|_{0, \Gamma_{c}} \\
& +\left\|\boldsymbol{\lambda}_{t}-\boldsymbol{\pi}_{H t} \boldsymbol{\lambda}_{t}\right\|_{0, \Gamma_{c}}\left\|\boldsymbol{u}_{t}-\boldsymbol{\pi}_{H t} \boldsymbol{u}_{t}\right\|_{0, \Gamma_{c}} \\
\leq & C H^{3 / 2},
\end{aligned}
$$

making use of (5.5) and (5.7).

(v) To estimate the last term, let us observe that the complementarity condition $\lambda_{n} u_{n}=0$ a.e. on $\Gamma_{c}$ yields:

$$
b\left(\boldsymbol{\lambda}_{H}-\boldsymbol{\lambda}, \boldsymbol{u}\right)=\int_{\Gamma_{c}} \lambda_{H n} u_{n} \mathrm{~d} \Gamma+\int_{\Gamma_{c}}\left(\boldsymbol{\lambda}_{H t}-\boldsymbol{\lambda}_{t}\right) \cdot \boldsymbol{u}_{t} \mathrm{~d} \Gamma .
$$

Since $u_{n} \leq 0$ and $\lambda_{H n} \geq 0$ a.e. on $\Gamma_{c}$, we have

$$
\int_{\Gamma_{c}} \lambda_{H n} u_{n} \mathrm{~d} \Gamma \leq 0
$$

Therefore

$$
b\left(\boldsymbol{\lambda}_{H}-\boldsymbol{\lambda}, \boldsymbol{u}\right) \leq \int_{\Gamma_{c}}\left(\boldsymbol{\lambda}_{H t}-\boldsymbol{\lambda}_{t}\right) \cdot \boldsymbol{u}_{t} \mathrm{~d} \Gamma .
$$

We now shall estimate (5.8). Noticing that

$$
\int_{\Gamma_{c}} \boldsymbol{\mu}_{H t} \cdot \boldsymbol{\psi}_{H} \mathrm{~d} \Gamma-\int_{\Gamma_{c}} s\left\|\boldsymbol{\psi}_{H}\right\| \mathrm{d} \Gamma \leq 0 \forall \boldsymbol{\psi}_{H} \in\left(L_{H}\right)^{2}
$$

and using that $-\boldsymbol{\lambda}_{t} \cdot \boldsymbol{u}_{t}+s\left\|\boldsymbol{u}_{t}\right\|=0$ a.e. on $\Gamma_{c}$ (see (3.4) and Th. 3.2), we have:

$$
\begin{aligned}
\int_{\Gamma_{c}}\left(\boldsymbol{\lambda}_{H t}-\boldsymbol{\lambda}_{t}\right) \cdot \boldsymbol{u}_{t} \mathrm{~d} \Gamma= & \int_{\Gamma_{c}}\left(\boldsymbol{\lambda}_{H t}-\boldsymbol{\lambda}_{t}\right) \cdot\left(\boldsymbol{u}_{t}-\boldsymbol{\pi}_{H t}\left(\boldsymbol{u}_{t}\right)\right) \mathrm{d} \Gamma+\int_{\Gamma_{c}}\left(\boldsymbol{\lambda}_{H t}-\boldsymbol{\lambda}_{t}\right) \cdot \boldsymbol{\pi}_{H t}\left(\boldsymbol{u}_{t}\right) \mathrm{d} \Gamma \\
& +\int_{\Gamma_{c}} \boldsymbol{\lambda}_{t} \cdot \boldsymbol{u}_{t} \mathrm{~d} \Gamma-\int_{\Gamma_{c}} s\left\|\boldsymbol{u}_{t}\right\| \mathrm{d} \Gamma \\
\leq & \int_{\Gamma_{c}}\left(\boldsymbol{\lambda}_{H t}-\boldsymbol{\lambda}_{t}\right) \cdot\left(\boldsymbol{u}_{t}-\boldsymbol{\pi}_{H t}\left(\boldsymbol{u}_{t}\right)\right) \mathrm{d} \Gamma+\int_{\Gamma_{c}} s\left(\left\|\boldsymbol{\pi}_{H t}\left(\boldsymbol{u}_{t}\right)\right\|-\left\|\boldsymbol{u}_{t}\right\|\right) \mathrm{d} \Gamma \\
& +\int_{\Gamma_{c}} \boldsymbol{\lambda}_{t} \cdot\left(\boldsymbol{u}_{t}-\boldsymbol{\pi}_{H t}\left(\boldsymbol{u}_{t}\right)\right) \mathrm{d} \Gamma \\
\leq & \int_{\Gamma_{c}}\left(\boldsymbol{\lambda}_{H t}-\boldsymbol{\lambda}_{t}\right) \cdot\left(\boldsymbol{u}_{t}-\boldsymbol{\pi}_{H t}\left(\boldsymbol{u}_{t}\right)\right) \mathrm{d} \Gamma+\int_{\Gamma_{c}} s\left(\left\|\boldsymbol{\pi}_{H t}\left(\boldsymbol{u}_{t}\right)-\boldsymbol{u}_{t}\right\|\right) \mathrm{d} \Gamma \\
& +\int_{\Gamma_{c}} \boldsymbol{\lambda}_{t} \cdot\left(\boldsymbol{u}_{t}-\boldsymbol{\pi}_{H t}\left(\boldsymbol{u}_{t}\right)\right) \mathrm{d} \Gamma \\
\leq & \left\|\boldsymbol{\lambda}_{H t}-\boldsymbol{\lambda}_{t}\right\|_{-\frac{1}{2}+\varepsilon, \Gamma_{c}}\left\|\boldsymbol{u}_{t}-\boldsymbol{\pi}_{H t}\left(\boldsymbol{u}_{t}\right)\right\|_{\frac{1}{2}-\varepsilon, \Gamma_{c}} \\
& +\|s\|_{0, \Gamma_{c}}\left\|\boldsymbol{u}_{t}-\boldsymbol{\pi}_{H t}\left(\boldsymbol{u}_{t}\right)\right\|_{0, \Gamma_{c}}+\left\|\boldsymbol{\lambda}_{t}\right\|_{0, \Gamma_{c}}\left\|\boldsymbol{u}_{t}-\boldsymbol{\pi}_{H t}\left(\boldsymbol{u}_{t}\right)\right\|_{0, \Gamma_{c}} \\
\leq & C H^{\frac{1}{2}+\varepsilon} \| \boldsymbol{\lambda}_{H}-\boldsymbol{\lambda}_{-\frac{1}{2}+\varepsilon, \Gamma_{c}}+C H
\end{aligned}
$$


making use of (5.5) and

$$
\left\|\boldsymbol{\mu}_{t}\right\|_{\left(H_{\Gamma_{c}}^{j}\right)^{2}} \leq\|\boldsymbol{\mu}\|_{\left(H_{\Gamma_{c}}^{j}\right)^{3}} \quad \text { for any } \boldsymbol{\mu}=\left(\mu_{n}, \boldsymbol{\mu}_{t}\right) \in \boldsymbol{H}_{\Gamma_{c}}^{j}, j= \pm \frac{1}{2}
$$

From (i)-(v) and the $\boldsymbol{V}$-ellipticity of the bilinear form $a(.,$.$) , we easily arrive at (5.2).$

The next lemma gives the error estimate for the Lagrange multiplier.

Lemma 5.3. Let all the assumptions of Lemma 5.2 together with (4.5) be satisfied. Then

$$
\begin{gathered}
\left\|\boldsymbol{\lambda}-\boldsymbol{\lambda}_{H}\right\|_{-\frac{1}{2}, \Gamma_{c}} \leq C\left\|\boldsymbol{u}-\boldsymbol{u}_{h}\right\|_{1, \Omega}+C H, \\
\left\|\boldsymbol{\lambda}-\boldsymbol{\lambda}_{H}\right\|_{-\frac{1}{2}+\varepsilon, \Gamma_{c}} \leq C H^{-\varepsilon}\left\|\boldsymbol{u}-\boldsymbol{u}_{h}\right\|_{1, \Omega}+C H^{1-\varepsilon},
\end{gathered}
$$

where $\varepsilon>0$ is arbitrarily small and $C$ is a positive constant which does not depend on $h$ and $H$.

Proof. Let us consider the equations in (3.6) and (4.3). Since $\boldsymbol{V}_{h} \subset \boldsymbol{V}$, we have

$$
\begin{aligned}
a\left(\boldsymbol{u}, \boldsymbol{v}_{h}\right)+b\left(\boldsymbol{\lambda}, \boldsymbol{v}_{h}\right) & =L\left(\boldsymbol{v}_{h}\right) & \forall \boldsymbol{v}_{h} \in \boldsymbol{V}_{h}, \\
a\left(\boldsymbol{u}_{h}, \boldsymbol{v}_{h}\right)+b\left(\boldsymbol{\lambda}_{H}, \boldsymbol{v}_{h}\right) & =L\left(\boldsymbol{v}_{h}\right) & \forall \boldsymbol{v}_{h} \in \boldsymbol{V}_{h} .
\end{aligned}
$$

Therefore

$$
a\left(\boldsymbol{u}-\boldsymbol{u}_{h}, \boldsymbol{v}_{h}\right)+b\left(\boldsymbol{\lambda}-\boldsymbol{\lambda}_{H}, \boldsymbol{v}_{h}\right)=0 \quad \forall \boldsymbol{v}_{h} \in \boldsymbol{V}_{h}
$$

and

$$
\begin{aligned}
b\left(\boldsymbol{\lambda}_{H}-\boldsymbol{\Pi}_{H} \boldsymbol{\lambda}, \boldsymbol{v}_{h}\right) & =a\left(\boldsymbol{u}-\boldsymbol{u}_{h}, \boldsymbol{v}_{h}\right)+b\left(\boldsymbol{\lambda}-\boldsymbol{\Pi}_{H} \boldsymbol{\lambda}, \boldsymbol{v}_{h}\right) \\
& \leq C\left\|\boldsymbol{u}-\boldsymbol{u}_{h}\right\|_{1, \Omega}\left\|\boldsymbol{v}_{h}\right\|_{1, \Omega}+C H\|\boldsymbol{\lambda}\|_{\frac{1}{2}, \Gamma_{c}}\left\|\boldsymbol{v}_{h}\right\|_{1, \Omega} \quad \forall \boldsymbol{v}_{h} \in \boldsymbol{V}_{h} .
\end{aligned}
$$

The inf-sup condition (4.5) yields:

$$
\tilde{\beta}\left\|\boldsymbol{\lambda}_{H}-\boldsymbol{\Pi}_{H} \boldsymbol{\lambda}\right\|_{-\frac{1}{2}, \Gamma_{c}} \leq \sup _{\boldsymbol{v}_{h} \in \boldsymbol{V}_{h}} \frac{b\left(\boldsymbol{\lambda}_{H}-\boldsymbol{\Pi}_{H} \boldsymbol{\lambda}, \boldsymbol{v}_{h}\right)}{\left\|\mid \boldsymbol{v}_{h}\right\|_{1, \Omega}} \leq C\left\|\boldsymbol{u}-\boldsymbol{u}_{h}\right\|_{1, \Omega}+C H\|\boldsymbol{\lambda}\|_{\frac{1}{2}, \Gamma_{c}} .
$$

Here we used the Korn's inequality and the fact that the dual norms \|\|$_{-\frac{1}{2}, \Gamma_{c}}$ and $\left\||\||_{-\frac{1}{2}, \Gamma_{c}}\right.$ are equivalent in $\boldsymbol{H}_{\Gamma_{c}}^{-\frac{1}{2}}$. The constant $\tilde{\beta}>0$ appearing on the left of $(5.12)$ is independent of $h$ and H.

The triangle inequality

$$
\left\|\boldsymbol{\lambda}-\boldsymbol{\lambda}_{H}\right\|_{-\frac{1}{2}, \Gamma_{c}} \leq\left\|\boldsymbol{\lambda}-\boldsymbol{\Pi}_{H} \boldsymbol{\lambda}\right\|_{-\frac{1}{2}, \Gamma_{c}}+\left\|\boldsymbol{\Pi}_{H} \boldsymbol{\lambda}-\boldsymbol{\lambda}_{H}\right\|_{-\frac{1}{2}, \Gamma_{c}}
$$

together with (5.12) and (5.5) proves (5.10).

Let us prove (5.11). The triangle and inverse inequality yield:

$$
\begin{aligned}
& \left\|\boldsymbol{\lambda}-\boldsymbol{\lambda}_{H}\right\|_{-\frac{1}{2}+\varepsilon, \Gamma_{c}} \leq\left\|\boldsymbol{\lambda}-\boldsymbol{\Pi}_{H} \boldsymbol{\lambda}_{H}\right\|_{-\frac{1}{2}+\varepsilon, \Gamma_{c}}+\left\|\boldsymbol{\Pi}_{H} \boldsymbol{\lambda}-\boldsymbol{\lambda}_{H}\right\|_{-\frac{1}{2}+\varepsilon, \Gamma_{c}} \\
& \quad \leq C\left(H^{1-\varepsilon}+H^{-\varepsilon}\left\|\boldsymbol{\Pi}_{H} \boldsymbol{\lambda}-\boldsymbol{\lambda}_{H}\right\|_{-\frac{1}{2}, \Gamma_{c}}\right),
\end{aligned}
$$

making use of (5.6). The rest of the proof now follows from (5.12)

We finally obtain the following global result giving an upper bound for our mixed finite element approximation. 
Theorem 5.1. Let $(\boldsymbol{u}, \boldsymbol{\lambda}),\left(\boldsymbol{u}_{h}, \boldsymbol{\lambda}_{H}\right)$ be the solution to (3.6), (4.3), respectively. Suppose that $\boldsymbol{u} \in \boldsymbol{H}^{2}(\Omega)$, the fourth order tensor A defining the Hooke's law is sufficiently smooth and the Ladyzhenskaya-Babuska-Brezzi condition (4.5) is satisfied. Then

$$
\left\|\boldsymbol{u}-\boldsymbol{u}_{h}\right\|_{1, \Omega}+\left\|\boldsymbol{\lambda}-\boldsymbol{\lambda}_{H}\right\|_{-\frac{1}{2}, \Gamma_{c}} \leq C H^{\frac{1}{2}}
$$

where the positive constant $C$ does not depend on $h$ and $H$.

Proof. Assembling (5.2), (5.10) and (5.11) we arrive at (5.13) making use that $h \leq C H$, where $C>0$ does not depend on $h$ and $H$.

Remark 5.1. Let us suppose that the slip bound $s$ is a positive constant. Then the error estimate (5.13) can be improved. Indeed, instead of (4.2) we define the set $\mathbf{M}_{H t}$ as follows:

$$
\mathbf{M}_{H t}=\left\{\boldsymbol{\mu}_{H t} \in\left(L_{H}\right)^{2} \mid \quad\left\|\boldsymbol{\mu}_{H t}\right\| \leq s \text { a.e. on } \Gamma_{c}\right\}
$$

Then $\mathbf{M}_{H t}$ is the internal approximation of $\mathbf{M}_{t}$ so that

$$
\int_{\Gamma_{c}} \boldsymbol{\mu}_{H t} \cdot \boldsymbol{\psi} \mathrm{d} \Gamma-s \int_{\Gamma_{c}}\|\boldsymbol{\psi}\| \mathrm{d} \Gamma \leq 0 \quad \forall \boldsymbol{\psi} \in \mathbf{L}^{2}\left(\Gamma_{c}\right) .
$$

It is very easy to verify that if $\boldsymbol{\mu}_{t} \in \mathbf{M}_{t}$ then $\boldsymbol{\pi}_{H t} \boldsymbol{\mu}_{t} \in \mathbf{M}_{H t}$ so that the estimates (i)-(iv) of Lemma 5.2 remain valid. Since $-\boldsymbol{\lambda}_{t} \cdot \boldsymbol{u}_{t}+s\left\|\boldsymbol{u}_{t}\right\|=0$ a.e. on $\Gamma_{c}$, the last term can be written as follows (see (5.8)):

$$
b\left(\boldsymbol{\lambda}_{H}-\boldsymbol{\lambda}, \boldsymbol{u}\right) \leq \int_{\Gamma_{c}}\left(\boldsymbol{\lambda}_{H t} \cdot \boldsymbol{u}_{t}-s\left\|\boldsymbol{u}_{t}\right\|\right) \mathrm{d} \Gamma \leq 0
$$

making use of (5.14) with $\boldsymbol{\mu}_{H t}:=\boldsymbol{\lambda}_{H t}$ and $\boldsymbol{\psi}:=\boldsymbol{u}_{t}$. Therefore this term which is responsible for the lower order of convergence can be omitted in (5.1). Under the assumptions of Theorem 5.1 we have

$$
\left\|\boldsymbol{u}-\boldsymbol{u}_{h}\right\|_{1, \Omega}+\left\|\boldsymbol{\lambda}-\boldsymbol{\lambda}_{H}\right\|_{-\frac{1}{2}, \Gamma_{c}} \leq C H^{\frac{3}{4}} .
$$

\section{Numerical REALizATion}

In this section we describe in brief the numerical method for the realization of $3 \mathrm{D}$ contact problems with given friction. This method is based on the the dual formulation of (3.5).

Let $\left(\boldsymbol{u}, \lambda_{n}, \boldsymbol{\lambda}_{t}\right)$ be the saddle-point of $\mathbf{L}$ on $\boldsymbol{V} \times M_{n} \times \mathbf{M}_{t}$ with the notation introduced in Section 2 . Then

$$
\begin{aligned}
\mathbf{L}\left(\boldsymbol{u}, \lambda_{n}, \boldsymbol{\lambda}_{t}\right) & =\min _{\boldsymbol{V}} \sup _{M_{n} \times \mathbf{M}_{t}} \mathbf{L}\left(\boldsymbol{v}, \mu_{n}, \boldsymbol{\mu}_{t}\right) \\
& =\max _{M_{n} \times \mathbf{M}_{t}} \inf _{\boldsymbol{V}} \mathbf{L}\left(\boldsymbol{v}, \mu_{n}, \boldsymbol{\mu}_{t}\right) .
\end{aligned}
$$

Denote

$$
\mathbf{S}\left(\mu_{n}, \boldsymbol{\mu}_{t}\right):=-\inf _{\boldsymbol{V}} \mathbf{L}\left(\boldsymbol{v}, \mu_{n}, \boldsymbol{\mu}_{t}\right)
$$

the dual functional. The dual variational formulation to (3.5) reads as follows:

$$
\left\{\begin{array}{c}
\text { Find }\left(\mu_{n}^{*}, \boldsymbol{\mu}_{t}^{*}\right) \in M_{n} \times \mathbf{M}_{t} \text { such that } \\
\mathbf{S}\left(\mu_{n}^{*}, \boldsymbol{\mu}_{t}^{*}\right) \leq \mathbf{S}\left(\mu_{n}, \boldsymbol{\mu}_{t}\right) \quad \forall\left(\mu_{n}, \boldsymbol{\mu}_{t}\right) \in M_{n} \times \mathbf{M}_{t} .
\end{array}\right.
$$

It is well-known [9] that (6.1) has a unique solution and, in addition $\left(\mu_{n}^{*}, \boldsymbol{\mu}_{t}^{*}\right)=\left(\lambda_{n}, \boldsymbol{\lambda}_{t}\right)$, where $\left(\lambda_{n}, \boldsymbol{\lambda}_{t}\right)$ is a part of the solution to (3.6). It is also readily seen [13] that $\mathbf{S}$ is a quadratic functional. Therefore (6.1) is a 
quadratic programming problem for the dual variable $\left(\mu_{n}, \boldsymbol{\mu}_{t}\right)$ defined on $\Gamma_{c}$ and subject to the linear, quadratic constraints $\mu_{n} \geq 0,\left\|\boldsymbol{\mu}_{t}\right\|^{2} \leq s^{2}$, respectively.

Let us recall that all constraints appearing in the dual formulation of $2 \mathrm{D}$ contact problems with given friction are linear [14]. This fact is important from the practical point of view: one can use [7] efficient CG type algorithms for solving (6.1). To be able to use the same algorithms in 3D problems, a linearization of the quadratic constraints will be necessary. This is what we shall do now.

Let $\mathbf{M}(r)$ be the ball in $\mathbb{R}^{2}$ of radius $r \geq 0$ with the center at origin:

$$
\mathbf{M}(r)=\left\{\vec{\lambda} \in \mathbb{R}^{2} \mid\|\vec{\lambda}\|^{2} \leq r^{2}\right\} .
$$

Next we construct a piecewise linear approximation of $\mathbf{M}(r)$ which will be realized by the intersection of $N$ squares rotated around the origin on a constant angle $\alpha=\pi /(2 N)$ :

$$
\mathbf{M}(r) \sim \cap_{i=1}^{N} \mathbf{M}_{i}(r),
$$

where

$$
\mathbf{M}_{i}(r)=\left\{\vec{\lambda} \in \mathbb{R}^{2} \mid\left\|\vec{\lambda}^{\alpha_{i}}\right\|_{\max } \leq r, \vec{\lambda}^{\alpha_{i}}:=\boldsymbol{m}_{\alpha_{i}} \vec{\lambda}\right\}
$$

with

$$
\boldsymbol{m}_{\alpha_{i}}=\left(\begin{array}{cc}
\cos \alpha_{i} & \sin \alpha_{i} \\
-\sin \alpha_{i} & \cos \alpha_{i}
\end{array}\right), \quad \alpha_{i}=i \alpha, \quad i=1, . ., N
$$

being the rotation matrix and $\|\vec{\lambda}\|_{\max }:=\max _{i=1,2}\left|\lambda_{i}\right|$.

Instead of (6.1) we shall consider the new problem:

$$
\left\{\begin{array}{c}
\text { Find }\left(\hat{\lambda}_{n}, \hat{\boldsymbol{\lambda}}_{t}\right) \in M_{n} \times \cap_{i=1}^{N} \mathbf{M}_{t}^{i} \text { such that } \\
\mathbf{S}\left(\hat{\lambda}_{n}, \hat{\boldsymbol{\lambda}}_{t}\right) \leq \mathbf{S}\left(\mu_{n}, \boldsymbol{\mu}_{t}\right) \quad \forall\left(\mu_{n}, \boldsymbol{\mu}_{t}\right) \in M_{n} \times \cap_{i=1}^{N} \mathbf{M}_{t}^{i},
\end{array}\right.
$$

where

$$
\mathbf{M}_{t}^{i}=\left\{\boldsymbol{\mu}_{t} \in \mathbf{L}^{2}\left(\Gamma_{c}\right), \mid \boldsymbol{\mu}_{t}(\boldsymbol{x}) \in \mathbf{M}_{i}(s(\boldsymbol{x})) \text { a.e. on } \Gamma_{c}\right\} .
$$

It is known (see [10]) that minimization problems on the intersection of convex sets can be transformed into a sequence of minimization problems over each subset of this intersection. In what follows we show how to proceed in our particular case.

We use the following notations:

$$
\begin{gathered}
\boldsymbol{\mu}^{i}:=\left(\mu_{n}, \boldsymbol{\mu}_{t}\right) \in M_{n} \times \mathbf{M}_{t}^{i}, \boldsymbol{\mu}=\left(\boldsymbol{\mu}^{1}, \ldots, \boldsymbol{\mu}^{N}\right) \in \prod_{i=1}^{N} M_{n} \times \mathbf{M}_{t}^{i}, \\
\mathbf{S}_{N}(\boldsymbol{\mu}):=\frac{1}{N} \sum_{i=1}^{N} \mathbf{S}\left(\boldsymbol{\mu}^{i}\right) .
\end{gathered}
$$

Then it is easy to see that (6.2) is equivalent to

$$
\left\{\begin{array}{cl} 
& \operatorname{Minimize} \mathbf{S}_{N}(\boldsymbol{\mu}) \\
\text { subject to } \quad \begin{array}{l}
\boldsymbol{\mu}^{i} \in M_{n} \times \mathbf{M}_{t}^{i}, \quad i=1, \ldots, N \\
\boldsymbol{\mu}^{i}=\boldsymbol{\mu}^{i+1}, \quad i=1, \ldots, N-1 .
\end{array}
\end{array}\right.
$$

Let us observe that the inclusion $\boldsymbol{\mu}^{i} \in M_{n} \times \mathbf{M}_{t}^{i}$ can be easily expressed by means of box constraints after an appropriate rotation of $\boldsymbol{\mu}^{i}$, i.e. we formally obtain the same situation as in $2 \mathrm{D}$ contact problems. The discretized version of (6.3) together with the augmented Lagrangian formulation has been used in (see [15]) for the numerical realization of 3D contact problems with friction, including Coulomb's model of friction. 


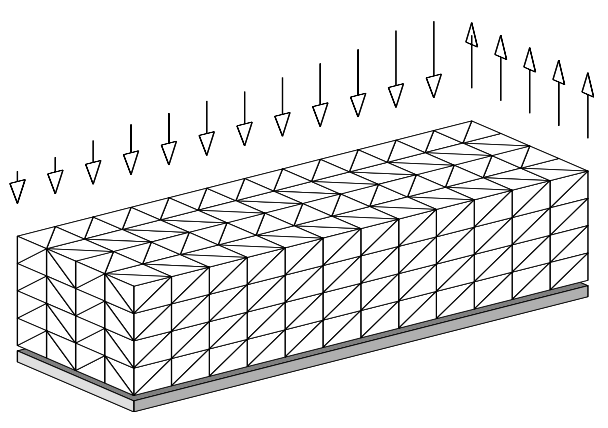

Figure 1. The loaded body.

The previous numerical method has a drawback, too, namely the number of variables is now equal to $N \times q$, where $N$ is the number of squares and $q=\operatorname{dim} \mathbf{L}_{H}$. This drawback however can be compensated by using coarser partitions of $\bar{\Gamma}_{c}$ when constructing $\mathbf{L}_{H}$ (another reason for the use of coarser partitions is mentioned in Lem. 4.1). It is worth noticing that one has to find a good compromise between the mesh sizes $h$ and $H$. Larger $H$ leads to a smaller number of the dual variables on the one hand, but to the poorer approximation of the non-penetration conditions on $\bar{\Gamma}_{c}$ on the other hand. In the following example the relative error between the "exact" and computed displacements for different ratios $H / h$ will be shown. By the "exact" solution we mean the displacement field computed by the same algorithm but using the "pointwise" Lagrange multipliers (algebraic multipliers) which act at each contact node.

The elastic body is represented by the brick $\Omega=(0,3) \times(0,1) \times(0,1)$ which is made of an elastic material characterized by Young modulus $E=21.19 \times 10^{10}$ and Poisson's ratio $\nu=0.277$. The body is fixed along the face $\Gamma_{\boldsymbol{u}}=\{0\} \times(0,1) \times(0,1)$ and surface tractions $\boldsymbol{g}$ act on $\Gamma_{\boldsymbol{g}}^{1}=(0,3) \times(0,1) \times\{1\}$ and $\Gamma_{\boldsymbol{g}}^{2}=\{3\} \times(0,1) \times(0,1)$, where

$$
\boldsymbol{g}=\left(0,0,-2 \times 10^{6} x_{1}\right) \text { on } \Gamma_{\boldsymbol{g}}^{1}
$$

and

$$
\boldsymbol{g}=\left(0,0,10^{6}\right) \text { on } \Gamma_{\boldsymbol{g}}^{2}
$$

The density of volume forces $\boldsymbol{f}$ is equal to zero and the slip bound $s=5.25 \times 10^{4}$ on $\Gamma_{c}$. The brick is cut into small cubes of size $h$ and each cube is divided into five tetrahedrons (see Fig. 1). Results for the ratios $H / h=2,4$ and the number of squares $N=2,4$ are summarized in Tables $1-4$. All computations were done on IBM SP2. From these results we see that even for higher ratios $H / h$ (i.e. for a less accurate satisfaction of non-penetration conditions) one can get a good approximation of the displacement field. In Table 5 the relative errors between the computed and "exact" solution for $n_{x}=36, n_{y}=n_{z}=12$ and $N=2$ on the first 5 grid levels above the contact surface are shown. The largest error occurs just on the contact (first line) surface while decreases when moving inside of the brick.

TABLE 1. Piecewise constant multipliers, $H / h=2, N=2$.

\begin{tabular}{|c||c|c|}
\hline$n_{x}, n_{y}, n_{z}$ & $n_{\lambda}$ & $e r r_{u}$ \\
\hline \hline $6,2,2$ & $9 \mathrm{~N}$ & 0.0202 \\
\hline $12,4,4$ & $36 \mathrm{~N}$ & 0.0178 \\
\hline $18,6,6$ & $81 \mathrm{~N}$ & 0.0064 \\
\hline $24,8,8$ & $144 \mathrm{~N}$ & 0.000691 \\
\hline $30,10,10$ & $225 \mathrm{~N}$ & 0.002201 \\
\hline $36,12,12$ & $324 \mathrm{~N}$ & 0.000685 \\
\hline $42,14,14$ & $441 \mathrm{~N}$ & 0.000323 \\
\hline
\end{tabular}




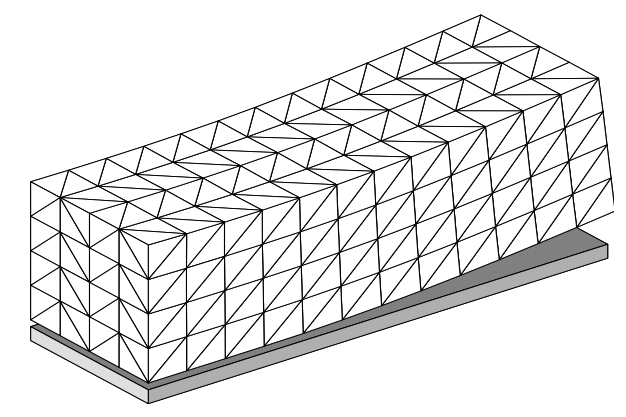

Figure 2. The body after deformation.

TABLE 2. Piecewise constant multipliers, $H / h=4, N=2$.

\begin{tabular}{|c||c|c|}
\hline$n_{x}, n_{y}, n_{z}$ & $n_{\lambda}$ & err $_{u}$ \\
\hline \hline $12,4,4$ & $9 \mathrm{~N}$ & 0.0107 \\
\hline $24,8,8$ & $36 \mathrm{~N}$ & 0.0249 \\
\hline $36,12,12$ & $54 \mathrm{~N}$ & 0.0045 \\
\hline
\end{tabular}

TABle 3. Piecewise constant multipliers, $H / h=2, N=4$.

\begin{tabular}{|c||c|c|}
\hline$n_{x}, n_{y}, n_{z}$ & $n_{\lambda}$ & $e r r_{u}$ \\
\hline \hline $6,2,2$ & $9 \mathrm{~N}$ & 0.0201 \\
\hline $12,4,4$ & $36 \mathrm{~N}$ & 0.0179 \\
\hline $18,6,6$ & $81 \mathrm{~N}$ & 0.0064 \\
\hline $24,8,8$ & $144 \mathrm{~N}$ & 0.000699 \\
\hline $30,10,10$ & $225 \mathrm{~N}$ & 0.002203 \\
\hline $36,12,12$ & $324 \mathrm{~N}$ & 0.000677 \\
\hline
\end{tabular}

TABle 4. Piecewise constant multipliers, $H / h=4, N=4$.

\begin{tabular}{|c||c|c|}
\hline$n_{x}, n_{y}, n_{z}$ & $n_{\lambda}$ & err $_{u}$ \\
\hline \hline $12,4,4$ & $9 \mathrm{~N}$ & 0.0106 \\
\hline $24,8,8$ & $36 \mathrm{~N}$ & 0.0249 \\
\hline $36,12,12$ & $54 \mathrm{~N}$ & 0.0044 \\
\hline
\end{tabular}

- $n_{x}, n_{y}, n_{z} \ldots$ the numbers of cubes of size $h$ in the $x, y, z$-directions;

- $n_{u} \ldots$ the number of the primal variables;

- $n_{\lambda} \ldots$ the number of the dual variables;

- $N$.. the number of squares approximating the circle;

- $\operatorname{err}_{u} \ldots$ the Euclidean norm of the relative error of the primal variable. 
TABLE 5. The relative error on different levels of the finite element mesh.

\begin{tabular}{|c||c|}
\hline$H / h=2$ & $H / h=4$ \\
\hline \hline 0.00083178 & 0.004496 \\
\hline 0.00066490 & 0.004283 \\
\hline 0.00062992 & 0.004184 \\
\hline 0.00061946 & 0.004132 \\
\hline 0.00061324 & 0.004097 \\
\hline
\end{tabular}

Acknowledgements. This research was supported by the project 2002-026-01 of the program Barrande. The first author acknowledges also the support of MSM 11320007. The authors wish to express their gratitude to R. Kučera who computed the previous example.

\section{REFERENCES}

[1] R.A. Adams, Sobolev Spaces. Academic Press (1975).

[2] G. Amontons, Sur l'origine de la résistance dans les machines. Mémoires de l'Académie Royale (1699) 206-222.

[3] L. Baillet and T. Sassi, Méthodes d'éléments finis avec hybridisation frontière pour les problèmes de contact avec frottement. C.R. Acad. Sci. Paris, Ser. I 334 (2002) 917-922.

[4] G. Bayada, M. Chambat, K. Lhalouani and T. Sassi, Éléments finis avec joints pour des problèmes de contact avec frottement de Coulomb non local. C.R. Acad. Sci. Paris, Ser. I 325 (1997) 1323-1328.

[5] P.-G. Ciarlet, The finite element method for elliptic problems, Handbook of Numerical Analysis, P.G. Ciarlet and J.L. Lions Eds., Vol. 2, Part 1, North-Holland (1991) 17-352.

[6] C.A. Coulomb, Théorie des machines simples. Mémoire de Mathématique et de Physique de l'Académie Royale 10 (1785) $145-173$.

[7] Z. Dostál, Box constrained quadratic programming with proportioning and projections. SIAM J. Opt. 7 (1997) 871-887.

[8] G. Duvaut and J.-L. Lions, Les inéquations en mécanique et en physique. Dunod, Paris (1972).

[9] I. Ekeland and R. Temam, Convex Analysis and Variational Problems. North-Holland, Amsterdam (1976).

[10] R. Glowinski, Numerical methods for nonlinear variational problems. Springer, New York (1984).

[11] P. Grisvard, Elliptic Problems in Nonsmooth Domains. Monogr. Studies Math., Pitman 24 (1985).

[12] J. Haslinger and I. Hlaváček, Approximation of the Signorini problem with friction by mixed finite element method, J. Math. Anal. Appl. 86 (1982) 99-122.

[13] J. Haslinger and P.D. Panagiolopoulas, Approximation of contact problems with friction by reciprocal variational formulations. Proc. Roy. Soc. Edinburgh 98A (1984) 365-383.

[14] J. Haslinger, I. Hlaváček and J. Nečas, Numerical methods for unilateral problems in solid mechanics, Handbook of Numerical Analysis, P.G. Ciarlet and J.L. Lions Eds., Vol. 4, Part 2, North-Holland (1996) 313-485.

[15] J. Haslinger, R. Kučera and Z. Dostál, An algorithm for numerical realization of 3D contact problems with Coulomb friction. J. Comput. Appl. Math. 164-165 (2004) 387-408.

[16] P. Hild, À propos d'approximation par éléments finis optimale pour les problèmes de contact unilatéral. C.R. Acad. Sci. Paris, Ser. I 326 (1998) 1233-1236.

[17] N. Kikuchi and J.T. Oden, Contact problems in elasticity: a study of variational inequalities and finite element methods. SIAM, Philadelphia (1988).

[18] D. Kinderlehrer and G. Stampacchia, An introduction to variational inequalities and their applications. Academic Press (1980).

[19] K. Lhalouani and T. Sassi, Nonconforming mixed variational formulation and domain decomposition for unilateral problems. East-West J. Numer. Math. 7 (1999) 23-30. 